\title{
Enriching datasets for sentiment analysis in tweets with instance selection
}

\author{
Eliseu Guimarães ${ }^{1,2}$, Daniela Vianna ${ }^{3}$, Aline Paes $^{1}$, Alexandre Plastino $^{1}$ \\ 1 Universidade Federal Fluminense, Brazil \\ 2 Marinha do Brasil \\ eliseuguimaraes@id.uff.br \{alinepaes,plastino\}@ic.uff.br \\ 3 Pesquisadora Independente \\ dvianna@gmail.com
}

\begin{abstract}
Sentiment analysis in tweets is a research field of great importance, mainly due to the popularity of Twitter. However, collecting and annotating tweets is an expensive and time-consuming task, making that some domains have only a limited set of labeled data. A promising strategy to handle this issue is to leverage labeled domains rich in data to select instances that enrich target datasets. This paper proposes different strategies for selecting instances from a set of labeled source datasets in order to improve the performance of classifiers trained only with the target dataset. Different approaches are proposed, including similarity metrics and variations in the number of selected instances. The results show that the size of the training set plays an essential role in the predictive capacity of the classifier. Furthermore, the results point out the importance of taking into account diversity criteria when selecting the instances.
\end{abstract}

CCS Concepts: • Computing methodologies $\rightarrow$ Transfer learning.

Keywords: machine learning, sentiment analysis, supervised learning, transfer learning

\section{INTRODUÇÃO}

A análise de sentimentos é o estudo computacional das opiniões, sentimentos, emoções e atitudes das pessoas [Liu 2020]. Com a crescente popularização das redes sociais, esse campo de pesquisa tem se tornado cada vez mais importante, visto que as pessoas são incentivadas a emitirem opiniões sobre os mais diversos assuntos. Uma dessas redes, o Twitter ${ }^{1}$, um serviço de microblog de textos curtos, chamados tweets, apresenta desafios próprios, como a presença de linguagem informal, a utilização de palavras grafadas de forma incorreta e a falta de contexto [Martínez-Cámara et al. 2014].

Uma das tarefas que a análise de sentimentos abrange é a deteç̧ão da polaridade de opiniões. No caso específico deste estudo, é tratada a detecção de polaridade em tweets. Abordagens baseadas em aprendizado de máquina são vastamente usadas para tratar essa tarefa, extraindo características dos tweets e as utilizando como atributos para o treinamento de classificadores. Em geral, dados de um determinado domínio são utilizados para treinar classificadores para o mesmo domínio. Contudo, há situações em que os dados rotulados disponíveis em um domínio não são suficientes para treinar um classificador com bom desempenho, seja devido ao fato de o domínio de interesse ser raro, ou por ser proibitivo rotular manualmente os dados existentes, ou ainda porque falta qualidade aos dados.

Para lidar com esse problema, uma abordagem oriunda da área de transferência de aprendizado [Pan and Yang 2010] é selecionar instâncias a partir de domínios-fonte para enriquecer o conjunto de

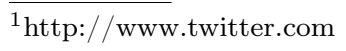

Copyright(C)2021. Permission to copy without fee all or part of the material printed in KDMiLe is granted provided that the copies are not made or distributed for commercial advantage, and that notice is given that copying is by permission of the Sociedade Brasileira de Computação. 
treinamento do classificador associado ao domínio-alvo, de modo a aumentar o seu desempenho preditivo [Guo et al. 2018; Liu et al. 2019; Ruder et al. 2017; Ruder and Plank 2017]. Porém, a maioria dos trabalhos anteriores requerem treinamento de métricas ou divisão das bases-fonte em subconjuntos. Além disso, os trabalhos anteriores não lidam especificamente com análise de sentimentos em tweets.

Este artigo investiga três abordagens de seleção de dados de um conjunto de bases-fonte oriundas de diversos domínios, com o objetivo de enriquecer o conjunto de treinamento para deteç̧ão de polaridade em uma base-alvo. No cenário investigado aqui, a base-alvo possui tweets rotulados para o treinamento de um classificador mas deseja-se melhorar o seu desempenho preditivo com um conjunto de dados enriquecido. Os resultados dos experimentos mostram que utilizar instâncias selecionadas de um conjunto de bases-fonte para enriquecer o conjunto de treinamento produz um aumento no desempenho dos classificadores em comparação com o treinamento apenas com a base-alvo. Esse aumento ocorre especialmente quando a seleção é composta de uma combinação de instâncias mais próximas e de instâncias mais distantes de cada instância da base-alvo.

O restante deste artigo se estrutura como segue. Na Seção 2, são apresentados trabalhos relacionados ao estudo desenvolvido, enquanto na Seção 3 é mostrada a metodologia utilizada por esta pesquisa. $\mathrm{Na}$ Seção 4, os resultados dos experimentos são apresentados, com suas respectivas conclusões sendo debatidas na Seção 5, onde ainda são apontados trabalhos futuros.

\section{TRABALHOS RELACIONADOS}

Diversos trabalhos têm proposto abordagens distintas para resolver o problema de seleção de dados de treinamento a partir de uma ou mais bases-fonte com o objetivo de treinar classificadores mais robustos para uma base-alvo de domínio distinto [Guo et al. 2018; Liu et al. 2019; Ruder et al. 2017; Ruder and Plank 2017]. Em [Guo et al. 2018], é utilizada uma abordagem do tipo mixture-of-experts, com diversas bases-fonte. Nesse trabalho, é considerado que cada base-fonte está alinhada a uma região distinta da base-alvo e uma métrica point-to-set é aprendida para ponderar os resultados de classificadores treinados com essas bases-fonte. O estudo conclui que as acurácias obtidas com esse tipo de estratégia foram superiores a se utilizar apenas uma base-fonte ou a união de todas as bases-fonte.

Por sua vez, [Liu et al. 2019] propõe uma abordagem de aprendizado por reforço, em que um framework, formado por dois componentes, busca instâncias relevantes e aprende melhores representações para elas. Um dos componentes é responsável por selecionar dados considerando um vetor de distribuição baseado na seleção de dados do passo anterior, enquanto o outro é responsável por fazer a extração de atributos dos dados, atualizar as recompensas para a geração do vetor de distribuição e gerar o classificador para a tarefa. Os resultados mostraram que esta abordagem teve um melhor desempenho em três de quatro bases-alvo utilizadas, em comparação com outros estudos.

Em [Ruder et al. 2017], são analisadas estratégias de seleção de dados, onde se consideram três fatores importantes para a seleção: a representação dos dados, a métrica de similaridade e o nível de seleção. Para cada uma das três representações avaliadas, é utilizada a métrica de similaridade mais comumente associada a ela. Os resultados apontam que utilizar seleção de subconjuntos de instâncias pode ter melhor desempenho preditivo do que a seleção individual de instâncias.

A abordagem proposta em [Ruder and Plank 2017] utiliza otimização Bayesiana para aprender uma métrica de similaridade de bases assumindo que diferentes tarefas e diferentes domínios pressupõem diferentes noções de similaridade. Foram utilizadas seis métricas de similaridade entre bases, três tipos de representações de dados e seis métricas de diversidade aplicadas ao conjunto de treinamento. $\mathrm{O}$ trabalho conclui que utilizar métricas de diversidade junto com métricas de similaridade melhora o desempenho preditivo, superando os resultados que selecionam aleatariamente dados ou usam uma única métrica.

Em comparação à literatura, neste estudo são apresentadas estratégias de seleção de dados que 
não requerem treinamento de métricas ou divisão das bases-fonte em subconjuntos. Adicionalmente, trata-se de um estudo específico de análise de sentimentos em tweets. Destaca-se ainda o fato de ser utilizado um grande e diverso conjunto de bases de diferentes domínios, o que confere robustez aos resultados.

\section{METODOLOGIA}

Nesta seção, está descrita a metodologia utilizada. Na Subseção 3.1, as bases utilizadas são descritas e são apresentados os procedimentos de pré-processamento executados para a extração dos atributos. A Subseção 3.2 descreve os procedimentos adotados na condução dos experimentos.

\subsection{Bases de dados e pré-processamento}

Nas avaliações conduzidas, utiliza-se um conjunto de 22 bases de dados de tweets em língua inglesa $^{2}$ [Carvalho and Plastino 2021]. As características dessas bases são apresentadas na Tabela I. Como pré-processamento dos tweets, inicialmente as menções a usuários e as URLs foram substituídas por expressões únicas. Os tweets foram, em seguida, tokenizados e colocados em letras minúsculas. Os atributos foram obtidos utilizando word embeddings, a partir de um modelo estático [Bravo-Marquez et al. 2016] que possui bom desempenho para análise de sentimentos em tweets [Carvalho and Plastino 2021]. O cálculo dos atributos de cada instância foi realizado computando a média dos embeddings referentes aos tokens da instância e, caso algum token não tivesse correspondência no modelo prétreinado, seus embeddings foram considerados como um vetor nulo.

\begin{tabular}{|c|c|c|c|c|c|c|c|c|c|c|c|}
\hline Base & Abreviação & \#pos & \#neg & $\%$ pos & Total & Base & Abreviação & \#pos & \#neg & $\%$ pos & Total \\
\hline irony & iro & 22 & 43 & $34 \%$ & 65 & archeage & arc & 724 & 994 & $42 \%$ & 1718 \\
\hline sarcasm & sar & 33 & 38 & $46 \%$ & 71 & SemEval18 & S18 & 865 & 994 & $47 \%$ & 1859 \\
\hline aisopos & ais & 159 & 119 & $57 \%$ & 278 & OMD & OMD & 710 & 1196 & $37 \%$ & 1906 \\
\hline SemEval15-Fig & S15 & 47 & 274 & $15 \%$ & 321 & $\mathrm{HCR}$ & $\mathrm{HCR}$ & 539 & 1369 & $28 \%$ & 1908 \\
\hline sentiment140 & sem & 182 & 177 & $51 \%$ & 359 & STS-gold & STS & 632 & 1402 & $31 \%$ & 2034 \\
\hline person & per & 312 & 127 & $71 \%$ & 439 & SentiStrength & SSt & 1340 & 949 & $59 \%$ & 2289 \\
\hline hobbit & hob & 354 & 168 & $68 \%$ & 522 & Target-dependent & Tar & 1734 & 1733 & $50 \%$ & 3467 \\
\hline iphone & iph & 371 & 161 & $70 \%$ & 532 & Vader & Vad & 2897 & 1299 & $69 \%$ & 4196 \\
\hline movie & mov & 460 & 101 & $82 \%$ & 561 & SemEval13 & S13 & 3183 & 1195 & $73 \%$ & 4378 \\
\hline sanders & san & 570 & 654 & $47 \%$ & 1224 & SemEval17 & S17 & 2375 & 3972 & $37 \%$ & 6347 \\
\hline Narr & nar & 739 & 488 & $60 \%$ & 1227 & SemEval16 & S16 & 8893 & 3323 & $73 \%$ & 12216 \\
\hline
\end{tabular}

Tabela I. Características das bases de dados.

\subsection{Procedimentos experimentais}

Nos experimentos realizados, foi utilizado o algoritmo SVM (Support Vector Machines), na sua implementação do scikit-learn [Pedregosa et al. 2011], com o parâmetro de ponderação de classe configurado para a forma balanceada devido a seu bom desempenho em análise de sentimentos em tweets [Barreto et al. 2021]. Além disso, foram considerados como baselines os valores de acurácia e de $F_{1}$ ponderados obtidos a partir de um procedimento de validação cruzada estratificada com 10 folds utilizando como conjunto de treinamento a própria base-alvo. Cada experimento foi realizado considerando cada uma das bases como base-alvo e as 21 restantes como a união das bases-fonte.

O primeiro experimento visava verificar se enriquecer o conjunto de treinamento com a maior quantidade possível de instâncias da união de bases-fonte, garantindo o balanceamento, produz melhora de desempenho em comparação com os baselines. Neste experimento, a base-alvo foi dividida nas mesmas 10 partições utilizadas para a geração dos valores baseline. Cada partição foi separada como teste e as nove restantes foram usadas como parte do conjunto de treinamento, que foi completado aleatoriamente com instâncias da união das bases-fonte. O modelo foi aplicado à partição de teste e

\footnotetext{
${ }^{2}$ https://github.com/joncarv/air-datasets
} 
o procedimento foi repetido para todas as partições, sendo calculadas a média da acurácia e do $F_{1}$ ponderado para se obter o desempenho final. A comparação com o baseline se dá pelo cálculo do ganho, computado como a razão entre o valor da métrica de desempenho obtida quando o conjunto de treinamento é formado pela base-alvo enriquecida pelas instâncias da união das bases-fonte e o valor do baseline.

O segundo experimento também considera a união das bases-fonte na construção do conjunto de treinamento. Entretanto, nesse experimento, diferentes métricas de seleção de instâncias são investigadas. O objetivo é identificar se existe um subconjunto da união das bases-fonte que, quando agregadas ao conjunto de treinamento, produz um classificador com poder preditivo superior ao baseline. Para este experimento, a base-alvo também foi dividida nas mesmas 10 partições utilizadas para a geração do baseline, sendo adotado um procedimento semelhante ao do experimento anterior. Porém, neste experimento, inicialmente é calculada a quantidade de instâncias que precisa ser agregada à classe minoritária das partições de treinamento para que o conjunto de treinamento fique balanceado. Considerando-se que esta quantidade vai ser retirada da união das bases-fonte, verifica-se qual será a classe minoritária do restante da união das bases-fonte e calcula-se a quantidade de instâncias dessa classe que deve ser agregada ao conjunto de treinamento, de forma a atender a um percentual de seleção.

São, então, adicionadas instâncias da união de bases-fonte segundo três critérios: (I) seleção aleatória de instâncias, (II) seleção das instâncias mais próximas a cada instância das partições que formam o conjunto de treinamento, (III) seleção das instâncias mais próximas e mais distantes a cada instância das partições que formam o conjunto de treinamento. Neste último critério, foram selecionadas quantidades iguais de instâncias mais próximas e instâncias mais distantes. Para os critérios (II) e (III), foi adotada como métrica de similaridade a distância Euclidiana. Ainda para estes dois últimos critérios, para cada instância das partições de treinamento da base-alvo só eram selecionadas instâncias da união da base-fonte que tivessem a mesma classe da instância da base-alvo. Para os três critérios, o balanceamento de classes foi sempre mantido. Os modelos gerados foram aplicados à partição de teste e seus resultados comparados com os baselines.

\section{RESULTADOS}

Nesta seção, são apresentados os resultados obtidos com os experimentos descritos na Seção 3. A Tabela II apresenta os resultados do primeiro experimento. Nela, são mostrados as acurácias e os $F_{1}$ ponderados obtidos quando o conjunto de treinamento é formado apenas pela base-alvo (colunas $A c_{a}$ e $F_{1-a}$ ) e quando ele é formado pela base-alvo em conjunto com a união das bases-fonte (colunas $A c_{a+f}$ e $F_{1-a+f}$ ). As colunas "Ganho Ac." e "Ganho $F_{1}$ " apresentam os ganhos de acurácia e $F_{1}$, isto é, os resultados das divisões das colunas $A c_{a+f}$ e $F_{1-a+f}$ pelas colunas $A c_{a}$ e $F_{1-a}$, respectivamente. Estão assinalados em negrito os valores de ganho maiores ou iguais a 1 , ou seja, aqueles valores que indicam que o desempenho utilizando a união das bases-fonte em conjunto com a base-alvo superou ou igualou o uso apenas da base-alvo. Tanto para a acurácia quanto para $F_{1}$ foram 15 as bases para as quais isso ocorreu. Cabe ressaltar ainda que, para a maioria das bases, os ganhos (razões) foram muito próximos a 1 , o que significa que a diferença de desempenho não foi significativa.

As Tabelas III-VI apresentam os resultados do segundo experimento. Na Tabela III, são apresentados os valores de acurácia e $F_{1}$ obtidos quando o conjunto de treinamento é formado por instâncias da base-alvo e instâncias da união das bases-fonte selecionadas de forma aleatória. O tamanho dessas seleções é definido percentualmente e, conforme pode ser observado na tabela, varia de $0,0 \%$ a 100,0\%, sendo este último percentual o equivalente ao experimento anterior.

A Tabela IV apresenta os ganhos obtidos considerando a seleção feita de forma aleatória, ou seja, os valores mostrados nesta tabela são os valores da Tabela III divididos pelos seus respectivos baselines, estando assinalados em negrito os casos em que o ganho é maior ou igual a 1. Nas três últimas 
Enriching datasets for sentiment analysis in tweets with instance selection

\begin{tabular}{|c|c|c|c|c|c|c|c|c|c|c|c|c|c|}
\hline Base & $A c_{a}$ & $A c_{a+f}$ & Ganho Ac. & $F_{1-a}$ & $F_{1-a+f}$ & Ganho $F_{1}$ & Base & $A c_{a}$ & $A c_{a+f}$ & Ganho Ac. & $F_{1-a}$ & $F_{1-a+f}$ & Ganho $F_{1}$ \\
\hline iro & 0,63 & 0,77 & 1,22 & 0,62 & 0,74 & 1,19 & sar & 0,69 & 0,85 & 1,22 & 0,67 & 0,85 & 1,27 \\
\hline ais & 0,94 & 0,95 & 1,00 & 0,94 & 0,95 & 1,00 & S15 & 0,90 & 0,76 & 0,84 & 0,90 & 0,78 & 0,87 \\
\hline sem & 0,87 & 0,87 & 1,01 & 0,87 & 0,87 & 1,01 & per & 0,78 & 0,82 & 1,06 & 0,79 & 0,82 & 1,05 \\
\hline hob & 0,89 & 0,83 & 0,93 & 0,89 & 0,83 & 0,93 & iph & 0,79 & 0,78 & 0,98 & 0,80 & 0,79 & 0,99 \\
\hline mov & 0,83 & 0,86 & 1,05 & 0,83 & 0,87 & 1,04 & san & 0,83 & 0,84 & 1,00 & 0,83 & 0,84 & 1,00 \\
\hline nar & 0,88 & 0,91 & 1,04 & 0,88 & 0,91 & 1,04 & $\operatorname{arc}$ & 0,87 & 0,85 & 0,99 & 0,87 & 0,85 & 0,98 \\
\hline S18 & 0,83 & 0,83 & 1,00 & 0,83 & 0,83 & 1,00 & OMD & 0,84 & 0,81 & 0,96 & 0,84 & 0,80 & 0,96 \\
\hline HCR & 0,75 & 0,78 & 1,04 & 0,76 & 0,75 & 0,99 & STS & 0,86 & 0,86 & 0,99 & 0,86 & 0,86 & 1,00 \\
\hline SSt & 0,80 & 0,82 & 1,02 & 0,80 & 0,82 & 1,02 & Tar & 0,83 & 0,82 & 0,98 & 0,83 & 0,82 & 0,98 \\
\hline Vad & 0,87 & 0,88 & 1,00 & 0,88 & 0,88 & 1,00 & S13 & 0,81 & 0,84 & 1,05 & 0,81 & 0,84 & 1,03 \\
\hline S17 & 0,88 & 0,88 & 1,01 & 0,88 & 0,88 & 1,00 & S16 & 0,85 & 0,86 & 1,02 & 0,85 & 0,86 & 1,02 \\
\hline
\end{tabular}

Tabela II. Acurácias e $F_{1}$ obtidos com a base-alvo (a) e base-alvo+base-fonte $(\mathrm{a}+\mathrm{f})$, com seus respectivos ganhos.

\begin{tabular}{|c|c|c|c|c|c|c|c|c|c|c|c|c|c|c|c|c|c|c|c|c|}
\hline \multirow{3}{*}{ Base } & \multicolumn{10}{|c|}{ Acurácia } & \multirow{2}{*}{\multicolumn{10}{|c|}{$\begin{array}{c}F_{1} \\
\text { Percentuais selecio }\end{array}$}} \\
\hline & \multicolumn{10}{|c|}{ Percentuais selecionados } & & & & & & & & & & \\
\hline & 0,0 & 0,5 & 1,0 & 2,5 & 5,0 & 10,0 & 20,0 & 40,0 & 80,0 & 100,0 & 0,0 & 0,5 & 1,0 & 2,5 & 5,0 & 10,0 & 20,0 & 40,0 & 80,0 & 100,0 \\
\hline iro & 0,65 & 0,66 & 0,66 & 0,72 & 0,77 & 0,74 & 0,75 & 0,75 & 0,77 & 0,77 & 0,64 & 0,64 & 0,64 & 0,71 & 0,74 & 0,71 & 0,73 & 0,73 & 0,74 & 0,74 \\
\hline sar & 0,69 & 0,79 & 0,76 & 0,75 & 0,75 & 0,82 & 0,78 & 0,86 & 0,85 & 0,85 & 0,67 & 0,78 & 0,75 & 0,74 & 0,73 & 0,81 & 0,77 & 0,85 & 0,84 & 0,85 \\
\hline ais & 0,93 & 0,92 & 0,92 & 0,91 & 0,92 & 0,92 & 0,94 & 0,94 & 0,94 & 0,95 & 0,93 & 0,92 & 0,92 & 0,91 & 0,92 & 0,92 & 0,94 & 0,94 & 0,94 & 0,95 \\
\hline S15 & 0,88 & 0,88 & 0,88 & 0,87 & 0,87 & 0,85 & 0,83 & 0,79 & 0,76 & 0,76 & 0,89 & 0,88 & 0,88 & 0,87 & 0,87 & 0,85 & 0,84 & 0,80 & 0,78 & 0,78 \\
\hline sem & 0,87 & 0,87 & 0,87 & 0,87 & 0,88 & 0,88 & 0,89 & 0,87 & 0,87 & 0,87 & 0,87 & 0,87 & 0,87 & 0,87 & 0,88 & 0,88 & 0,89 & 0,87 & 0,87 & 0,87 \\
\hline per & 0,77 & 0,79 & 0,80 & 0,80 & 0,82 & 0,81 & 0,81 & 0,82 & 0,82 & 0,82 & 0,77 & 0,79 & 0,80 & 0,80 & 0,82 & 0,81 & 0,81 & 0,82 & 0,82 & 0,82 \\
\hline hob & 0,88 & 0,88 & 0,88 & 0,86 & 0,87 & 0,84 & 0,83 & 0,83 & 0,83 & 0,83 & 0,88 & 0,88 & 0,88 & 0,86 & 0,86 & 0,84 & 0,83 & 0,83 & 0,82 & 0,83 \\
\hline iph & 0,80 & 0,80 & 0,81 & 0,81 & 0,79 & 0,78 & 0,79 & 0,78 & 0,78 & 0,78 & 0,80 & 0,80 & 0,82 & 0,82 & 0,80 & 0,79 & 0,80 & 0,79 & 0,79 & 0,79 \\
\hline mov & 0,84 & 0,87 & 0,86 & 0,85 & 0,84 & 0,86 & 0,85 & 0,86 & 0,86 & 0,86 & 0,83 & 0,86 & 0,85 & 0,85 & 0,84 & 0,86 & 0,85 & 0,86 & 0,86 & 0,87 \\
\hline san & 0,84 & 0,84 & 0,84 & 0,84 & 0,83 & 0,83 & 0,83 & 0,83 & 0,83 & 0,84 & 0,84 & 0,84 & 0,84 & 0,84 & 0,83 & 0,83 & 0,83 & 0,83 & 0,83 & 0,84 \\
\hline nar & 0,88 & 0,89 & 0,89 & 0,89 & 0,89 & 0,90 & 0,90 & 0,91 & 0,91 & 0,91 & 0,88 & 0,89 & 0,89 & 0,89 & 0,90 & 0,90 & 0,90 & 0,91 & 0,91 & 0,91 \\
\hline arc & 0,87 & 0,87 & 0,87 & 0,87 & 0,87 & 0,86 & 0,85 & 0,86 & 0,86 & 0,85 & 0,87 & 0,87 & 0,87 & 0,87 & 0,86 & 0,86 & 0,85 & 0,86 & 0,86 & 0,85 \\
\hline S18 & 0,83 & 0,83 & 0,83 & 0,84 & 0,84 & 0,83 & 0,83 & 0,83 & 0,83 & 0,83 & 0,83 & 0,83 & 0,83 & 0,84 & 0,84 & 0,83 & 0,83 & 0,83 & 0,83 & 0,83 \\
\hline OMD & 0,83 & 0,83 & 0,83 & 0,83 & 0,83 & 0,84 & 0,82 & 0,82 & 0,81 & 0,81 & 0,83 & 0,82 & 0,83 & 0,83 & 0,83 & 0,83 & 0,82 & 0,81 & 0,81 & 0,80 \\
\hline $\mathrm{HCR}$ & 0,79 & 0,79 & 0,79 & 0,80 & 0,80 & 0,79 & 0,79 & 0,79 & 0,79 & 0,78 & 0,77 & 0,77 & 0,77 & 0,77 & 0,77 & 0,76 & 0,76 & 0,76 & 0,76 & 0,75 \\
\hline STS & 0,86 & 0,87 & 0,87 & 0,87 & 0,87 & 0,87 & 0,87 & 0,87 & 0,86 & 0,8 & 0,86 & 0,87 & 0,87 & 0,87 & 0,8 & 0,8 & 0,87 & 0,87 & 0,86 & 0,86 \\
\hline SSt & 0,81 & 0,81 & 0,81 & 0,81 & 0,81 & 0,81 & 0,82 & 0,81 & 0,82 & 0,82 & 0,81 & 0,81 & 0,81 & 0,81 & 0,81 & 0,81 & 0,82 & 0,81 & 0,82 & 0,82 \\
\hline Tar & 0,83 & 0,83 & 0,83 & 0,83 & 0,83 & 0,83 & 0,82 & 0,82 & 0,82 & 0,82 & 0,83 & 0,83 & 0,83 & 0,83 & 0,83 & 0,83 & 0,82 & 0,82 & 0,82 & 0,82 \\
\hline Vad & 0,87 & 0,87 & 0,88 & 0,88 & 0,88 & 0,88 & 0,88 & 0,87 & 0,88 & 0,88 & 0,88 & 0,88 & 0,88 & 0,88 & 0,88 & 0,88 & 0,88 & 0,88 & 0,88 & 0,88 \\
\hline $\mathrm{S} 13$ & 0,82 & 0,83 & 0,83 & 0,83 & 0,84 & 0,84 & 0,84 & 0,84 & 0,85 & 0,84 & 0,83 & 0,83 & 0,83 & 0,83 & 0,84 & 0,84 & 0,84 & 0,84 & 0,84 & 0,84 \\
\hline S17 & 0,88 & 0,88 & 0,88 & 0,88 & 0,88 & 0,88 & 0,89 & 0,89 & 0,89 & 0,88 & 0,88 & 0,88 & 0,88 & 0,88 & 0,88 & 0,88 & 0,89 & 0,88 & 0,88 & 0,88 \\
\hline S16 & 0,85 & 0,86 & 0,86 & 0,86 & 0,86 & 0,86 & 0,86 & 0,86 & 0,86 & 0,86 & 0,85 & 0,86 & 0,86 & 0,86 & 0,86 & 0,86 & 0,86 & 0,86 & 0,86 & 0,86 \\
\hline
\end{tabular}

Tabela III. Acurácias e $F_{1}$ obtidos com seleção aleatória de percentuais da base-fonte associados à base-alvo.

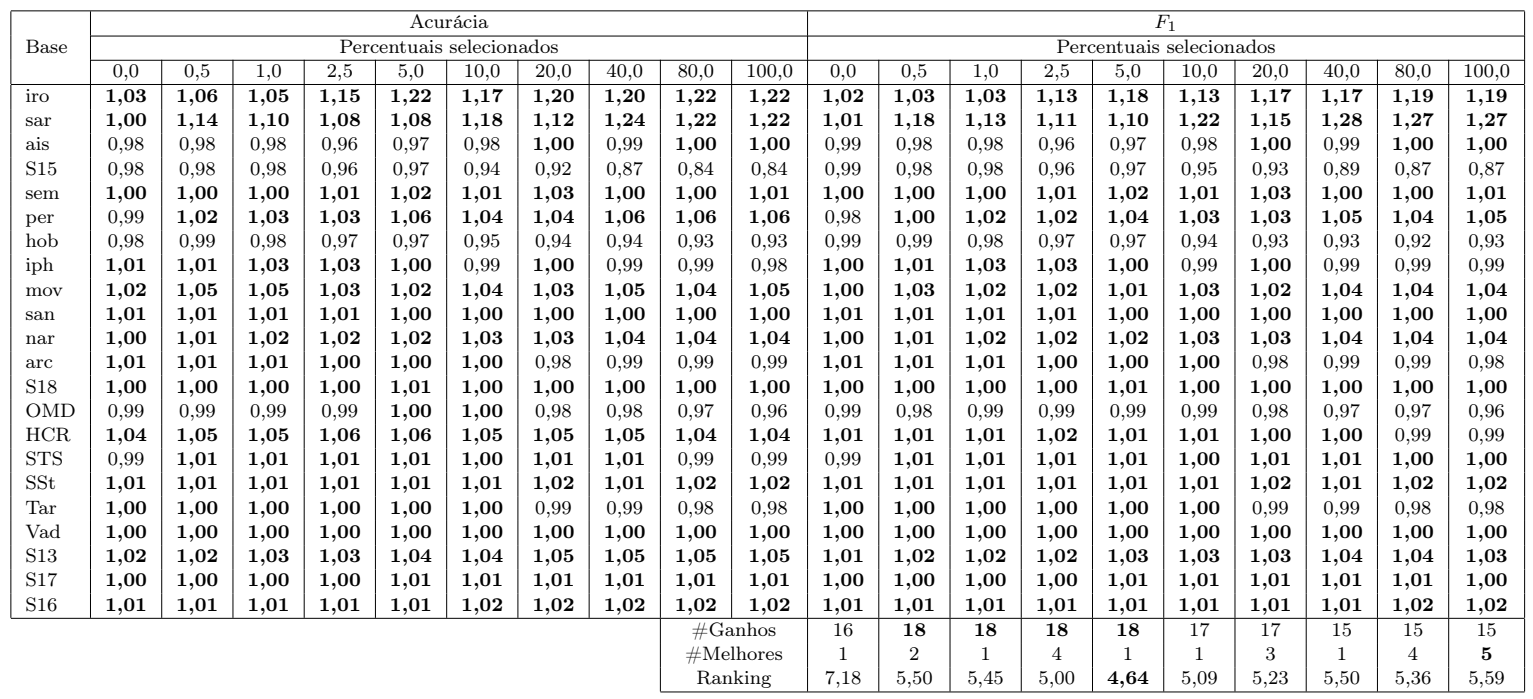

Tabela IV. Ganhos de acurácia e $F_{1}$ obtidos com seleção aleatória de percentuais da base-fonte associados à base-alvo.

linhas da tabela, são colocados, para cada percentual selecionado, as quantidades de ganhos de $F_{1}$ que são maiores ou iguais a 1 (\#Ganhos), a quantidade de vezes que o percentual produziu o melhor desempenho em termos de $F_{1}$ dentre todos os percentuais analisados (\#Melhores) e o ranking do percentual. Considerando apenas os valores de \#Ganhos, a melhor seleção ocorreu com 0,5\%, 1,0\%, 
$2,5 \%$ e $5,0 \%$ da união das bases-fonte (18 ganhos), seguidas pela seleção de 10,0\% e 20,0\% (17 ganhos). No que diz respeito ao desempenho dos melhores ganhos (\#Melhores), o melhor percentual foi 100,0\% (5 melhores), seguido por 2,5\% e 80,0\% (4 melhores). Selecionar 5,0\% da união das bases-fonte teve a melhor posição média no ranking (posição média de 4,64). Como nenhum percentual se mostrou claramente melhor, pode ser considerado que $2,5 \%, 5,0 \%$ e 100,0\% tiveram o melhor desempenho geral para essa estratégia.

A Tabela $\mathrm{V}$ apresenta os resultados dos ganhos de acurácia e $F_{1}$ para a estratégia que seleciona as instâncias da união das bases-fonte pelo critério da proximidade por distância Euclidiana a cada uma das instâncias do conjunto de treinamento da base-alvo. Por questões de limitação de espaço, não será apresentada a tabela com os valores absolutos de acurácia e $F_{1}$. Com esta abordagem, as maiores quantidades de ganhos ocorreram com a seleção de 10,0\% (19 ganhos) e 5,0\% (18 ganhos) e, considerando o critério de melhores resultados, o percentual selecionado com melhor desempenho foi $20,0 \%$ (6 melhores). Este percentual também obteve a melhor posição média no ranking $(3,23)$, e podemos considerar que a seleção de $10,0 \%$ ou $20,0 \%$ são as melhores para esta estratégia.

\begin{tabular}{|c|c|c|c|c|c|c|c|c|c|c|c|c|c|c|c|c|c|c|c|c|}
\hline \multirow{3}{*}{ Base } & \multicolumn{10}{|c|}{ Acurácia } & \multicolumn{10}{|c|}{$F_{1}$} \\
\hline & \multicolumn{10}{|c|}{ Percentuais selecionados } & \multicolumn{10}{|c|}{ Percentuais selecionados } \\
\hline & 0,0 & 0,5 & 1,0 & 2,5 & 5,0 & 10,0 & 20,0 & 40,0 & 80,0 & 100,0 & 0,0 & 0,5 & 1,0 & 2,5 & 5,0 & 10,0 & 20,0 & 40,0 & 80,0 & 100,0 \\
\hline iro & 1,15 & 1,20 & 1,18 & 1,18 & 1,15 & 1,20 & 1,23 & 1,20 & 1,25 & 1,33 & 1,15 & 1,19 & 1,15 & 1,16 & 1,13 & 1,18 & 1,22 & 1,17 & 1,23 & 1,32 \\
\hline sar & 1,00 & 1,06 & 1,08 & 1,12 & 1,12 & 1,12 & 1,18 & 1,16 & 1,18 & 1,22 & 1,01 & 1,08 & 1,11 & 1,16 & 1,16 & 1,16 & 1,23 & 1,21 & 1,21 & 1,26 \\
\hline S15 & 0,96 & 0,97 & 0,96 & 0,94 & 0,94 & 0,92 & 0,90 & 0,89 & 0,84 & 0,81 & 0,97 & 0,98 & 0,96 & 0,95 & 0,95 & 0,93 & 0,92 & 0,91 & 0,87 & 0,85 \\
\hline sem & 1,01 & 1,01 & 1,00 & 1,01 & 1,01 & 1,01 & 1,02 & 1,02 & 0,99 & 0,98 & 1,01 & 1,01 & 1,00 & 1,01 & 1,01 & 1,01 & 1,02 & 1,02 & 0,99 & 0,98 \\
\hline per & 1,01 & 1,01 & 1,01 & 1,03 & 1,03 & 1,07 & 1,05 & 1,04 & 1,06 & 1,07 & 1,00 & 1,00 & 1,00 & 1,02 & 1,03 & 1,06 & 1,04 & 1,03 & 1,05 & 1,05 \\
\hline hob & 0,99 & 0,99 & 0,98 & 0,97 & 0,97 & 0,96 & 0,96 & 0,95 & 0,92 & 0,92 & 0,99 & 0,99 & 0,98 & 0,97 & 0,97 & 0,95 & 0,96 & 0,94 & 0,92 & 0,91 \\
\hline iph & 0,99 & 1,01 & 1,01 & 1,01 & 1,03 & 1,03 & 1,04 & 1,03 & 0,99 & 0,98 & 0,99 & 1,01 & 1,01 & 1,01 & 1,03 & 1,02 & 1,04 & 1,03 & 0,99 & 0,98 \\
\hline mov & 1,00 & 1,01 & 1,02 & 1,03 & 1,03 & 1,05 & 1,05 & 1,04 & 1,05 & 1,05 & 1,00 & 1,00 & 1,01 & 1,01 & 1,02 & 1,04 & 1,04 & 1,04 & 1,04 & 1,04 \\
\hline san & 1,01 & 1,00 & 1,01 & 1,00 & 1,01 & 1,00 & 1,01 & 1,00 & 0,99 & 0,97 & 1,01 & 1,00 & 1,01 & 1,01 & 1,01 & 1,00 & 1,01 & 1,00 & 0,99 & 0,97 \\
\hline nar & 1,00 & 1,00 & 1,00 & 1,01 & 1,01 & 1,02 & 1,03 & 1,03 & 1,01 & 1,02 & 1,00 & 1,00 & 1,00 & 1,01 & 1,01 & 1,02 & 1,03 & 1,02 & 1,01 & 1,02 \\
\hline arc & 1,00 & 1,00 & 0,99 & 0,99 & 1,00 & 1,00 & 0,99 & 0,99 & 0,97 & 0,97 & 1,00 & 1,00 & 0,99 & 0,99 & 1,00 & 1,00 & 0,99 & 0,99 & 0,97 & 0,97 \\
\hline S18 & 1,00 & 1,01 & 1,00 & 1,01 & 1,00 & 1,00 & 1,01 & 1,01 & 1,00 & 1,01 & 1,00 & 1,01 & 1,00 & 1,01 & 1,00 & 1,00 & 1,01 & 1,01 & 1,00 & 1,01 \\
\hline OMD & 0,97 & 0,97 & 0,98 & 0,99 & 0,99 & 0,98 & 0,98 & 0,97 & 0,96 & 0,95 & 0,97 & 0,97 & 0,98 & 0,99 & 0,99 & 0,98 & 0,98 & 0,96 & 0,95 & 0,94 \\
\hline $\mathrm{HCR}$ & 1,02 & 1,03 & 1,02 & 1,04 & 1,04 & 1,04 & 1,04 & 1,05 & 1,04 & 1,04 & 1,00 & 1,00 & 1,00 & 1,00 & 1,00 & 1,00 & 1,00 & 1,00 & 1,00 & 1,00 \\
\hline STS & 0,99 & 1,00 & 1,00 & 1,00 & 1,00 & 1,00 & 1,01 & 0,99 & 0,98 & 0,97 & 0,99 & 1,00 & 1,00 & 1,00 & 1,00 & 1,00 & 1,01 & 0,99 & 0,98 & 0,97 \\
\hline SSt & 1,00 & 1,00 & 1,00 & 1,01 & 1,02 & 1,02 & 1,02 & 1,02 & 1,01 & 1,01 & 1,00 & 1,00 & 1,00 & 1,01 & 1,02 & 1,02 & 1,02 & 1,01 & 1,01 & 1,01 \\
\hline Tar & 1,00 & 1,00 & 1,00 & 1,00 & 1,00 & 1,00 & 1,00 & 0,98 & 0,98 & 0,98 & 1,00 & 1,00 & 1,00 & 1,00 & 1,00 & 1,00 & 1,00 & 0,98 & 0,98 & 0,98 \\
\hline Vad & 0,99 & 0,99 & 0,99 & 0,99 & 1,00 & 1,00 & 1,00 & 1,00 & 1,00 & 1,00 & 0,99 & 0,99 & 0,99 & 0,99 & 1,00 & 1,00 & 1,00 & 1,00 & 1,00 & 1,00 \\
\hline $\mathrm{S} 13$ & 1,01 & 1,01 & 1,02 & 1,02 & 1,02 & 1,02 & 1,03 & 1,03 & 1,04 & 1,04 & 1,01 & 1,01 & 1,01 & 1,01 & 1,02 & 1,02 & 1,02 & 1,02 & 1,02 & 1,02 \\
\hline S17 & 1,00 & 1,00 & 1,00 & 1,00 & 1,00 & 1,00 & 1,01 & 1,01 & 1,00 & 1,00 & 1,00 & 1,00 & 1,00 & 1,00 & 1,00 & 1,00 & 1,01 & 1,01 & 1,00 & 1,00 \\
\hline $\mathrm{S} 16$ & 1,01 & 1,01 & 1,01 & 1,01 & 1,01 & 1,01 & 1,02 & 1,02 & 1,02 & 1,02 & 1,00 & 1,00 & 1,00 & 1,01 & 1,01 & 1,01 & 1,01 & 1,01 & 1,02 & 1,01 \\
\hline & & & & & & & & & $\# 0$ & hos & 15 & 17 & 16 & 16 & 18 & 19 & 17 & 15 & 12 & 12 \\
\hline & & & & & & & & & $\# \mathrm{M}$ & & 2 & 1 & 0 & 2 & 1 & 3 & 6 & 3 & 2 & 2 \\
\hline & & & & & & & & & & king & 7,36 & 6,05 & 6,73 & 5,05 & 4,64 & 3,95 & 3,23 & 4,77 & 6,41 & 6,50 \\
\hline
\end{tabular}

Tabela V. Ganhos de acurácia e $F_{1}$ obtidos com seleção percentual das instâncias da base-fonte mais próximas à basealvo.

Na Tabela VI, são apresentados os ganhos de acurácia e $F_{1}$ obtidos com a estratégia de selecionar as instâncias da união das bases-fonte que sejam mais próximas e as mais distantes a cada instância do conjunto de treinamento da base-alvo pelo critério da distância Euclidiana. Para esta estratégia, os melhores resultados para o critério de \#Ganhos foram obtidos selecionando 1,0\% e 2,5\% (19 ganhos) e 10,0\% e 20,0\% (18 ganhos). Para \#Melhores os melhores resultados foram produzidos selecionando $40,0 \%$ e 100,0\% (5 melhores), o que explica o fato de 40,0\% ter obtido a melhor posição média no ranking $(3,86)$, sendo considerado o melhor percentual para esta estratégia. Um detalhe importante a considerar é que, como são selecionadas por esta estratégia quantidades iguais de instâncias mais próximas e mais distantes, selecionar 40,0\% da união das bases-fonte é simplesmente acrescentar as $20,0 \%$ instâncias mais distantes na união das bases-fonte às $20,0 \%$ mais próximas que representam um dos melhores resultados da estratégia anterior.

Na Tabela VII, estão comparados os melhores resultados para cada uma das estratégias. Estão assinalados em negrito, novamente, valores de ganho maiores ou iguais a 1. As três últimas linhas são geradas levando em consideração apenas as seis combinações de estratégia-percentual colocadas nesta tabela. No que diz respeito ao número de ganhos maiores ou iguais a 1, a seleção das instâncias mais próximas com um percentual de 10,0\% apresentou o melhor resultado (19 ganhos), seguida de 


\begin{tabular}{|c|c|c|c|c|c|c|c|c|c|c|c|c|c|c|c|c|c|c|c|c|}
\hline Base & \multicolumn{10}{|c|}{ Acurácia } & \multicolumn{10}{|c|}{$F_{1}$} \\
\hline iro & 1,03 & 1,13 & 1,20 & 1,20 & 1,28 & 1,30 & 1,23 & 1,25 & 1,25 & 1,25 & 1,00 & 1,12 & 1,21 & 1,21 & 1,27 & 1,30 & 1,23 & 1,24 & 1,21 & 1,21 \\
\hline sar & 0,96 & 1,02 & 1,10 & 1,10 & 1,10 & 1,12 & 1,20 & 1,14 & 1,20 & 1,24 & 0,97 & 1,04 & 1,12 & 1,13 & 1,14 & 1,15 & 1,25 & 1,18 & 1,25 & 1,29 \\
\hline S15 & 1,00 & 0,99 & 0,98 & 0,95 & 0,96 & 0,94 & 0,91 & 0,88 & 0,84 & 0,82 & 0,99 & 0,99 & 0,98 & 0,96 & 0,97 & 0,95 & 0,92 & 0,90 & 0,87 & 0,85 \\
\hline sem & 1,01 & 1,00 & 1,03 & 1,01 & 1,02 & 1,03 & 1,01 & 1,03 & 1,02 & 1,01 & 1,01 & 1,00 & 1,03 & 1,01 & 1,02 & 1,03 & 1,01 & 1,03 & 1,02 & 1,01 \\
\hline per & 1,04 & 1,01 & 1,02 & 1,03 & 1,03 & 1,05 & 1,06 & 1,05 & 1,06 & 1,06 & 1,03 & 1,00 & 1,01 & 1,02 & 1,02 & 1,04 & 1,05 & 1,04 & 1,04 & 1,05 \\
\hline hob & 0,98 & 0,99 & 1,00 & 0,98 & 0,96 & 0,98 & 0,95 & 0,96 & 0,93 & 0,92 & 0,98 & 0,99 & 1,00 & 0,98 & 0,96 & 0,97 & 0,95 & 0,96 & 0,93 & 0,90 \\
\hline nar & 1,00 & 1,00 & 1,01 & 1,01 & 1,02 & 1,02 & 1,03 & 1,04 & 1,04 & 1,04 & 1,00 & 1,00 & 1,01 & 1,01 & 1,02 & 1,02 & 1,03 & 1,04 & 1,04 & 1,04 \\
\hline $\operatorname{arc}$ & 1,00 & 0,99 & 1,00 & 1,00 & 0,99 & 0,99 & 1,00 & 0,99 & 0,99 & 0,99 & 1,00 & 0,99 & 1,00 & 1,00 & 0,99 & 0,99 & 1,00 & 0,99 & 0,99 & 0,99 \\
\hline S18 & 1,00 & 1,00 & 1,00 & 1,00 & 1,00 & 1,00 & 1,01 & 1,01 & 1,00 & 1,00 & 1,00 & 1,00 & 1,00 & 1,00 & 1,00 & 1,00 & 1,01 & 1,01 & 1,00 & 1,00 \\
\hline OMD & 0,98 & 0,99 & 0,99 & 1,00 & 0,99 & 1,00 & 0,98 & 0,98 & 0,97 & 0,96 & 0,98 & 0,99 & 0,99 & 1,00 & 0,99 & 1,00 & 0,98 & 0,97 & 0,96 & 0,96 \\
\hline $\mathrm{HCR}$ & 1,03 & 1,04 & 1,04 & 1,03 & 1,03 & 1,04 & 1,05 & 1,05 & 1,05 & 1,05 & 1,01 & 1,01 & 1,01 & 1,00 & 1,00 & 1,00 & 1,01 & 1,01 & 1,00 & 1,00 \\
\hline STS & 1,01 & 1,01 & 1,00 & 1,01 & 1,02 & 1,02 & 1,01 & 1,01 & 0,99 & 0,98 & 1,00 & 1,01 & 1,00 & 1,01 & 1,02 & 1,02 & 1,01 & 1,01 & 1,00 & 0,99 \\
\hline SSt & 1,00 & 1,00 & 1,01 & 1,02 & 1,02 & 1,02 & 1,02 & 1,02 & 1,02 & 1,03 & 1,00 & 1,00 & 1,01 & 1,02 & 1,02 & 1,02 & 1,02 & 1,02 & 1,02 & 1,02 \\
\hline & & & & & & & & & & & 16 & 17 & 19 & 19 & 17 & 18 & 18 & 16 & 17 & 16 \\
\hline & & & & & & & & & $\# \mathrm{Me}$ & hores & 1 & 0 & 2 & 2 & 0 & 2 & 1 & 5 & 4 & 5 \\
\hline & & & & & & & & & Ran & & 7,05 & 7,09 & 6,18 & 6,00 & 5,36 & 4,00 & 4,50 & 3,86 & 4,77 & 5,73 \\
\hline
\end{tabular}

Tabela VI. Ganhos de acurácia e $F_{1}$ obtidos com seleção percentual das instâncias da base-fonte mais próximas e mais distantes à base-alvo.

perto por selecionar aleatoriamente com um percentual de 2,5\% ou 5,0\% (18 ganhos). Levando em consideração o critério da quantidade de vezes que o percentual produziu os melhores resultados, selecionar as mais próximas com um percentual de $20,0 \%$ ou as mais próximas e as mais distantes com um percentual de $40,0 \%$ obteve os melhores resultados ( 5 ganhos), sendo que este último percentual também obteve o melhor desempenho no ranking médio $(2,82)$.

\begin{tabular}{|l|c|c|c|c|c|c|}
\hline \multirow{2}{*}{ Base } & \multicolumn{3}{|c|}{ Aleatória } & \multicolumn{2}{c|}{ Próximas } & Próximas e distantes \\
\cline { 2 - 7 } & $2,5 \%$ & $5,0 \%$ & $100,0 \%$ & $10,0 \%$ & $20,0 \%$ & $40,0 \%$ \\
\hline iro & $\mathbf{1 , 1 3}$ & $\mathbf{1 , 1 8}$ & $\mathbf{1 , 1 9}$ & $\mathbf{1 , 1 8}$ & $\mathbf{1 , 2 2}$ & $\mathbf{1 , 2 4}$ \\
sar & $\mathbf{1 , 1 1}$ & $\mathbf{1 , 1 0}$ & $\mathbf{1 , 2 7}$ & $\mathbf{1 , 1 6}$ & $\mathbf{1 , 2 3}$ & $\mathbf{1 , 1 8}$ \\
ais & 0,96 & 0,97 & $\mathbf{1 , 0 0}$ & $\mathbf{1 , 0 0}$ & 0,99 & 0,99 \\
S15 & 0,96 & 0,97 & 0,87 & 0,93 & 0,92 & 0,90 \\
sem & $\mathbf{1 , 0 1}$ & $\mathbf{1 , 0 2}$ & $\mathbf{1 , 0 1}$ & $\mathbf{1 , 0 1}$ & $\mathbf{1 , 0 2}$ & $\mathbf{1 , 0 3}$ \\
per & $\mathbf{1 , 0 2}$ & $\mathbf{1 , 0 4}$ & $\mathbf{1 , 0 5}$ & $\mathbf{1 , 0 6}$ & $\mathbf{1 , 0 4}$ & $\mathbf{1 , 0 4}$ \\
hob & 0,97 & 0,97 & 0,93 & 0,95 & 0,96 & 0,96 \\
iph & $\mathbf{1 , 0 3}$ & $\mathbf{1 , 0 0}$ & 0,99 & $\mathbf{1 , 0 2}$ & $\mathbf{1 , 0 4}$ & $\mathbf{1 , 0 2}$ \\
mov & $\mathbf{1 , 0 2}$ & $\mathbf{1 , 0 1}$ & $\mathbf{1 , 0 4}$ & $\mathbf{1 , 0 4}$ & $\mathbf{1 , 0 4}$ & $\mathbf{1 , 0 4}$ \\
san & $\mathbf{1 , 0 1}$ & $\mathbf{1 , 0 0}$ & $\mathbf{1 , 0 0}$ & $\mathbf{1 , 0 0}$ & $\mathbf{1 , 0 1}$ & $\mathbf{1 , 0 1}$ \\
nar & $\mathbf{1 , 0 2}$ & $\mathbf{1 , 0 2}$ & $\mathbf{1 , 0 4}$ & $\mathbf{1 , 0 2}$ & $\mathbf{1 , 0 3}$ & $\mathbf{1 , 0 4}$ \\
arc & $\mathbf{1 , 0 0}$ & $\mathbf{1 , 0 0}$ & 0,98 & $\mathbf{1 , 0 0}$ & 0,99 & 0,99 \\
S18 & $\mathbf{1 , 0 0}$ & $\mathbf{1 , 0 1}$ & $\mathbf{1 , 0 0}$ & $\mathbf{1 , 0 0}$ & $\mathbf{1 , 0 1}$ & $\mathbf{1 , 0 1}$ \\
OMD & 0,99 & 0,99 & 0,96 & 0,98 & 0,98 & 0,97 \\
HCR & $\mathbf{1 , 0 2}$ & $\mathbf{1 , 0 1}$ & 0,99 & $\mathbf{1 , 0 0}$ & $\mathbf{1 , 0 0}$ & $\mathbf{1 , 0 1}$ \\
STS & $\mathbf{1 , 0 1}$ & $\mathbf{1 , 0 1}$ & $\mathbf{1 , 0 0}$ & $\mathbf{1 , 0 0}$ & $\mathbf{1 , 0 1}$ & $\mathbf{1 , 0 1}$ \\
SSt & $\mathbf{1 , 0 1}$ & $\mathbf{1 , 0 1}$ & $\mathbf{1 , 0 2}$ & $\mathbf{1 , 0 2}$ & $\mathbf{1 , 0 2}$ & $\mathbf{1 , 0 2}$ \\
Tar & $\mathbf{1 , 0 0}$ & $\mathbf{1 , 0 0}$ & 0,98 & $\mathbf{1 , 0 0}$ & $\mathbf{1 , 0 0}$ & 0,99 \\
Vad & $\mathbf{1 , 0 0}$ & $\mathbf{1 , 0 0}$ & $\mathbf{1 , 0 0}$ & $\mathbf{1 , 0 0}$ & $\mathbf{1 , 0 0}$ & $\mathbf{1 , 0 0}$ \\
S13 & $\mathbf{1 , 0 2}$ & $\mathbf{1 , 0 3}$ & $\mathbf{1 , 0 3}$ & $\mathbf{1 , 0 2}$ & $\mathbf{1 , 0 2}$ & $\mathbf{1 , 0 3}$ \\
S17 & $\mathbf{1 , 0 0}$ & $\mathbf{1 , 0 1}$ & $\mathbf{1 , 0 0}$ & $\mathbf{1 , 0 0}$ & $\mathbf{1 , 0 1}$ & $\mathbf{1 , 0 1}$ \\
S16 & $\mathbf{1 , 0 1}$ & $\mathbf{1 , 0 1}$ & $\mathbf{1 , 0 2}$ & $\mathbf{1 , 0 1}$ & $\mathbf{1 , 0 1}$ & $\mathbf{1 , 0 1}$ \\
\hline \#Ganhos & 18 & 18 & 15 & $\mathbf{1 9}$ & 17 & 16 \\
\#Melhores & 4 & 4 & 4 & 2 & $\mathbf{5}$ & $\mathbf{5}$ \\
Ranking & 3,77 & 3,36 & 4,05 & 3,95 & 2,91 & $\mathbf{2 , 8 2}$ \\
\hline
\end{tabular}

Tabela VII. Comparação entre os melhores percentuais para ganho de $F_{1}$ em todas as estratégias.

\section{CONCLUSÕES E TRABALHOS FUTUROS}

Neste artigo, investigou-se se utilizar dados de bases-fonte proporciona um aumento no desempenho de modelos de classificação para uma base-alvo rotulada, no contexto de análise de sentimentos em tweets. Para isto, foram desenvolvidos dois experimentos, o primeiro que agrega a totalidade da união das bases-fonte ao conjunto de treinamento do classificador e o segundo propondo estratégias de seleção de instâncias dessa união de bases-fonte de acordo com três estratégias: (I) seleção aleatória de 
instâncias, (II) seleção das instâncias mais próximas a cada instância das partições de treinamento da base-alvo e (III) seleção das instâncias mais próximas e mais distantes de cada instância das partições de treinamento da base-alvo. Para todos os experimentos, o conjunto de treinamento era balanceado e os modelos gerados foram testados em partições da base-alvo por meio de validação cruzada. Os resultados foram comparados com o desempenho do classificador treinado apenas com a base-alvo em termos de acurácia e $F_{1}$ ponderado por intermédio do cálculo do ganho - divisão entre os valores das métricas usando a união de bases-fonte com a base-alvo e usando somente a base-alvo.

Os resultados do primeiro experimento mostraram que aproveitar um conjunto de bases-fonte para compor o conjunto de treinamento produz ganhos de desempenho para a maioria das bases-alvo, tanto em termos de acurácia quanto em termos de $F_{1}$. No entanto, esse ganho não se mostrou elevado para a maioria das bases, o que indicou que alguma estratégia de seleção de instâncias poderia ser útil.

Os resultados do segundo experimento apontaram que algumas combinações de estratégia e percentual apresentaram bom desempenho. Para a seleção aleatória, os melhores desempenhos ocorreram selecionando $2,5 \%, 5,0 \%$ e 100,0\% . Considerando a seleção das instâncias mais próximas a cada instância das partições de treinamento da base-alvo, selecionar $10,0 \%$ ou $20,0 \%$ obteve o melhor resultado, ao passo que para a estratégia que inclui selecionar também as mais distantes os melhores resultados foram encontrados com a seleção de $40,0 \%$ da união das bases-fonte. Entre essas seis combinações de estratégia-percentual, a que apresentou o melhor resultado geral foi a seleção de 40,0\% com a estratégia das mais próximas e das mais distantes. Estes resultados indicam que utilizar uma união de bases-fonte para ser agregada ao conjunto de treinamento de classificadores para uma base-alvo pode trazer ganhos de desempenho, em particular porque esse conjunto de bases-fonte pode ser usado para ampliar, balancear e diversificar o conjunto de treinamento.

Trabalhos futuros incluem a utilização de outras métricas para a seleção de instâncias. Adicionalmente, um parâmetro que regule a proporção de instâncias mais próximas e mais distantes a serem utilizadas pode ser acrescentado e ajustado.

\section{REFERENCES}

Barreto, S., Moura, R., Carvalho, J., Paes, A., and Plastino, A. Sentiment analysis in tweets: an assessment study from classical to modern text representation models. CoRR vol. abs/2105.14373, 2021.

Bravo-Marquez, F., Frank, E., Mohammad, S. M., and Pfahringer, B. Determining word-emotion associations from tweets by multi-label classification. In Proceedings of the 2016 IEEE/WIC/ACM Int. Conf. on Web Intelligence (WI). IEEE, pp. 536-539, 2016.

Carvalho, J. and Plastino, A. On the evaluation and combination of state-of-the-art features in twitter sentiment analysis. Artificial Intelligence Review vol. 54, pp. 1887-1936, 03, 2021.

Guo, J., Shah, D., And Barzilay, R. Multi-source domain adaptation with mixture of experts. In Proceedings of the 2018 Conference on Empirical Methods in Natural Language Processing. ACL, pp. 4694-4703, 2018.

Liu, B. Sentiment Analysis: Mining Opinions, Sentiments, and Emotions. Studies in Natural Language Processing. Cambridge University Press, 2020.

Liu, M., Song, Y., Zou, H., And ZHAng, T. Reinforced training data selection for domain adaptation. In Proceedings of the 57th Annual Meeting of the Association for Computational Linguistics. ACL, pp. 1957-1968, 2019.

Martínez-Cámara, E., Martín-Valdivia, M., López, L., and Montejo-RÁez, A. Sentiment analysis in twitter. Natural Language Engineering vol. 20, pp. 1-28, 01, 2014.

Pan, S. J. And YAng, Q. A Survey on Transfer Learning. IEEE Transactions on Knowledge and Data Engineering 22 (10): 1345-1359, 2010.

Pedregosa, F., Varoquaux, G., Gramfort, A., Michel, V., Thirion, B., Grisel, O., Blondel, M., Prettenhofer, P., Weiss, R., Dubourg, V., Vanderplas, J., Passos, A., Cournapeau, D., Brucher, M., Perrot, M., and Duchesnay, E. Scikit-learn: Machine learning in Python. Journal of Machine Learning Research vol. 12, pp. 2825-2830, 2011.

Ruder, S., Ghaffari, P., And Breslin, J. G. Data selection strategies for multi-domain sentiment analysis. CoRR vol. abs/1702.02426, 2017.

Ruder, S. And Plank, B. Learning to select data for transfer learning with Bayesian Optimization. In Proceedings of the 2017 Conference on Empirical Methods in Natural Language Processing. ACL, pp. 372-382, 2017. 